\title{
Electronic Differential And Neuro-Fuzzy Sliding Mode Control With Extended State Observer For An Electric Vehicle System
}

\author{
Ibrahim Farouk Bouguenna, ${ }^{1, *}$, Ahmed Azaiz ${ }^{1}$, Ahmed Tahour $^{2}$, Ahmed Larbaoui $^{2}$ \\ ${ }^{1}$ RCAM Laboratory, University of Sidi Bel-Abbes, 22000, Algeria \\ ${ }^{2}$ University of Mascara, 29000, Algeria
}

\begin{abstract}
In this paper a neuro-fuzzy-sliding mode control (NFSMC) with extended state observer (ESO) technique; is designed to guarantee the traction of an electric vehicle with two distinct permanent magnet synchronous motor (PMSM). Each PMSM systems ( source-convertermotor) are attached to an electronic differential (ED),in order to adjust the senses of direction of the vehicle and sustain a stable speed by adapting the difference in velocity of each motor-wheel according to the direction in the case of a turn. Two types of controllers are employed by a hybrid control scheme to assure the control and the performance of the vehicle. This hybrid control scheme guarantees the stability of the vehicle by ED, reduces the chattering phenomena in the PMSM electric motor, and improves the disturbance rejection ability which employs tow types of controllers. The neuro-fuzzy sliding mode control on the direct current loop and ESO controller on the speed loop, and the quadratic current loop; taking into account the dynamic of the vehicle. Simulation runs under Matlab/Simulink to assess the efficiency , and strength of the recommended control method on the closed loop system.
\end{abstract}

\section{Introduction}

The intense use of electric car in the world has played a very important role in increasing the purity of the air in overcrowded areas. Limiting the impact of air polluted by the means of transport on humanity , and has decreased the global warming of the earth [1].

To achieve cleaner and greener mobility in highly urbanized cities around the world, the utilization of electric vehicle must be considered as one of the solutions [2]. Many researches on electric vehicles has been carried out such as control system which is a very important part of the vehicle or electric vehicle's brain. It determines the total performance of the vehicle, as it is the key to improve motor efficiency. in our work a permanent magnet synchronous motor has been adopted as the electric vehicle (EV) propulsion.

Many researches has been done on electric vehicles testing the control of its traction chain, which is translated into the control of these engines wheels. As a result, The performance-dependent process of the engine-wheel assembly system is distinguished by

* Corresponding author: faroukspvusto@yahoo.fr 
the smooth spin throughout the engine speed orbit, total torque control at zero speed, and rapid acceleration and deceleration.

On a standard vehicle the drive wheels are joined by half-shafts restrained by a bevel gear. On each of the wheels there is a torque equal to half of that provided by the return angle. Because of the hard connection arranged with the drive shaft, the two drive wheels revolve at the same angular speed, which has no specific disadvantage in walking in a straight line [3]. The track of the inner wheel is decreased contrasted to that of the outer wheel; For these two distances to be realized in the same time interval, it is essential that the angular speed of the two wheels is diverse. If the wheels are not driving, no problem, besides it is vital to insert a differential mechanism permitting the wheels to spin at dissimilar speeds. There is a system in classic vehicles that assures the difference in speeds while ensuring torque for the two driving wheels [4]; named mechanical differential. In this work we will substitute the mechanical differential with an electronic differential in order to lessen the drive line components, therefore enhancing the general dependability and efficiency. This option will also diminish the drive line weight since mechanical differential and gear reduction are not used. But, one of the main problems in the construction of these EVs (without mechanical differential) is to assure the vehicle steadiness specially while cornering or under slippery road conditions [5]. This calls for a specific traction control system . The control of the traction effort transmitted by each wheel is at the base of the command strategies intending to enhance the steadfastness of the vehicle. Where the wheels are controlled independently by using an electric motorization.

With in particular context the electric vehicle (EV) propulsion has been adopted by permanent magnet synchronous motor. These motors (PMSMs) have comprehensive industrial applications containing electric vehicles, robotics, and wind energy conversion systems as cited in [6].

This can be imputed to desirable performance characteristics comprising high power density, high torque to weight ratio, high reliability and high efficiency [6]. Many control techniques for PMSMs have been developed in literature studies. Although its poor performance the PI controller remains a popular choice because of its simplicity, and ease of implementation, it does not take disturbances and uncertainties into account, leading to poor performance [7].

In consequence of the improvement that have been made to develop the digital signal processor technology, power electronics, control theories, more and more advanced control methods that are introduced to the PMSM control problem, e.g., adaptive control [8], robust control [9], sliding mode control [10], neural network control [11], and fuzzy control [12], etc. Which can ameliorate the electric vehicle's performance founded on the PMSM from different aspects.

Due to its ability to reject internal parameter variations and external disturbances, the sliding mode control is a popular control. SMC has found extensive applications in the areas of power electronics and electric machines [13]. As a result, SMC has been widely and successfully applied into the position and velocity control of PMSM. However, SMC like anything else has its own negatives, i.e., chattering phenomenon, which has originated from the interaction between parasitic dynamics and high frequency switching control [14]. For that, the control method was proposed to ensure the traction of the electric vehicle in particular to eliminate the chattering phenomenon, which is the neuro-fuzzy-sliding mode control (RNFSMC) with extended state observer (ESO). The hybrid neuro-fuzzy sliding mode control with extended state observer [15] is one of the most common controls that has associated several techniques: fuzzy control [16] and sliding mode control [17], neural network, and disturbances rejection laws to employ the advantages of all the techniques to restrain the disadvantages of regulation by conventional adjustment algorithms and to 
improve the performance of the controller system (stability, accuracy, speed, robustness, etc...).

The FIS forms are a useful computing framework based on the concepts of fuzzy set theory, fuzzy if-then rules and fuzzy reasoning. The ANFIS [21] is a FIS implemented in the framework of an adaptive fuzzy neural network. Combining the explicit knowledge representation of a FIS with the learning power of ANNs. Usually, the transformation of human knowledge into a fuzzy system (in the form of rules and membership functions) does not give the target response accurately. So, the parameters of the FIS should be determined optimally [24].

The lumped disturbances of the system which are composed of internal dynamics and external disturbances are considered as new state of the system by Extended State Observer (ESO) [18]. Where both states and lumped disturbances can be estimated by this observer. Based on the ESO, a feed forward compensation for the disturbances can also be employed in the control design.

In this paper, we try to explain the development of a robust control of an electric vehicle system, that helps to guarantee a stability and performances of the vehicle using ED and adaptating the closed-loop combining the extended state observer (ESO) with the adaptive neuro-fuzzy inference system technique together.

The content of this paper is arranged as follows: section 2 reviews the principle components of the traction system and their model equations (vehicle dynamic, Electronic differential , electric motor model). Section 3 demonstrate the theory of control ( sliding mode control and Neuro-fuzzy-sliding mode control with disturbance rejection law model ). Section 4 a simulation results to confirm the validity of the proposed technique of control. Finally we conclude by a conclusion in section 5 .

\section{Electric Vehicle Traction System}

This section is dedicated to the dynamics of the electric vehicle and the different components on board the vehicle and their equations

\subsection{Vehicle dynamics}

Consider a vehicle of mass, $\mathrm{m}_{\mathrm{v}}$ proceeding at a velocity $\mathrm{v}$, up a slope of angle $\alpha$, as in Fig.1. The force propelling the vehicle forward $\mathrm{F}_{\mathrm{T}}$, the tractive effort, has to overcome the following forces (rolling resistance, aerodynamic resistance, gravitational force) [25]:

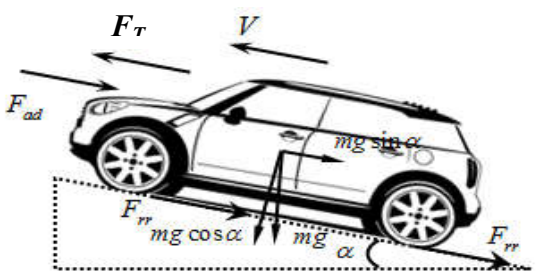

Fig.1 . Block diagram of the forces acting on the vehicle.

$$
\begin{gathered}
F_{r r}=C_{r r} \cdot m_{v} \cdot g \cdot \cos (\alpha) \\
F_{a d}=\frac{1}{2} \cdot \rho \cdot A_{f} \cdot C_{d} \cdot\left(v-v_{0}\right)^{2}
\end{gathered}
$$




$$
\begin{aligned}
& F_{g}= \pm m_{v} \cdot g \cdot \sin (\alpha) \\
& m_{v} \frac{d}{d t} V(t)=F_{T}(t)-\left(F_{a d}(t)+F_{r r}(t)+F_{g}(t)\right) \\
& F_{a c c}=m_{v} \cdot \frac{d}{d t} \cdot v(t)
\end{aligned}
$$

Where $\mathrm{C}_{\mathrm{rr}}$ is the coefficient of rolling resistance, $\rho$ density of the air, $\mathrm{v}_{0}$ the wind speed, $A_{f}$ the frontal surface of the vehicle, coefficient of penetration into the air which takes values $\mathrm{C}_{\mathrm{d}}$. and the force due to the acceleration $F_{a c c}$ ensures the dynamic behavior desired by the driver.

\subsection{Mathematical model of PMSM}

In this work we use the three phases induction motor type PMSM in the stationary (d q) reference frame, the mathematics mode of permanent-magnet synchronous motor is shown as below [20].

$$
\left\{\begin{array}{l}
i_{d}^{\bullet}=-\frac{R_{s}}{L_{d}} i_{d}+\frac{L_{q}}{L_{d}} p \omega_{r} i_{q}+\frac{1}{L_{d}} u_{d} \\
i_{q}^{\bullet}=-\frac{R_{s}}{L_{q}} i_{q}-\frac{L_{d}}{L_{q}} p \omega_{r} i_{d}-\frac{p \psi_{f}}{L_{q}} \omega_{r}+\frac{1}{L_{q}} u_{q} \\
\omega_{r}^{\bullet}=\frac{p\left(L_{d}-L_{q}\right) i_{d}+p \psi_{f}}{J} i_{q}-\frac{1}{J} C_{r}-\frac{f}{J} \omega_{r}
\end{array}\right.
$$

With $\mathrm{R}_{\mathrm{s}}$ :Stator resistance, $L_{d}, L_{q} \mathrm{~d}$ and $\mathrm{q}$ axis stator inductances $\psi_{\mathrm{f}}$ :Permanent-magnet flux linkage, $i_{d}, i_{q}$ : Stator currents, $u_{q}$ : Stator voltages, $\omega_{r}$ : Speed mechanic, $J$ : Moment of inertia, $f$ : Coefficient of viscous friction, $p$ : Number of pole pairs, $C_{r}:$ Load torque.

\subsection{Modelling of The Electronic Differential}

The proposed structure design propulsion system assures that over a straight trajectory of the wheels ability to roll exactly at the same speed by allowing one to develop an electronic differential. whereas in a curve trajectory; the difference between these two wheel's speed assures the vehicles trajectory over the curve. The fig. 2 represents the vehicle structure describing a curve, where $L$ represents the wheelbase, $\delta$ the steering angle, $d$ the distance between the wheels of the same axle and $\omega_{r_{-} L}$ and $\omega_{r_{-} R}$ the angular speeds of the left and right wheel drives, respectively.

In a curve the linear speed of each wheel drive is expressed as a function of the vehicle speed and the radius of curve:

$$
\begin{aligned}
& v_{L}=\omega_{v}\left(R+\frac{d}{2}\right) \\
& v_{R}=\omega_{v}\left(R-\frac{d}{2}\right)
\end{aligned}
$$

The curve radius is related to the wheelbase and steering angle:

$$
R=\frac{L}{\tan (\delta)}
$$


Substituting (9) into Equations (7) and (8), we obtain the angular speed in each wheel drive:

$$
\left\{\begin{array}{c}
\omega_{r L}=\frac{L+0.5 d \tan (\delta)}{L} \omega_{v} \\
\omega_{r R}=\frac{L-0.5 d \tan (\delta)}{L} \omega_{v}
\end{array}\right.
$$

The difference between the angular speeds of the wheel drives is expressed by the relation

$$
\Delta \omega=\omega_{r R}-\omega_{r L}=\frac{d \tan (\delta)}{L} \omega_{v}
$$

Numeric sign of the steering angle signal indicates the curve direction:

$$
\begin{aligned}
& \delta \succ 0 \Rightarrow \text { Turn right } \\
& \delta=0 \Rightarrow \text { Straight ahead } \\
& \delta \prec 0 \Rightarrow \text { Turn left. }
\end{aligned}
$$

When the vehicle begins a curve, the driver imposes a steering angle to the wheels. The electronic differential however acts immediately on the two motors reducing the speed of the inner wheel, increases the speed of the outer wheel. The driving wheel angular speeds are:

$$
\left\{\begin{array}{l}
\omega_{r_{-} L_{-} r e f}=\omega_{v}+\frac{\Delta \omega}{2} \\
\omega_{r_{-} R_{-} r e f}=\omega_{v}-\frac{\Delta \omega}{2}
\end{array}\right.
$$

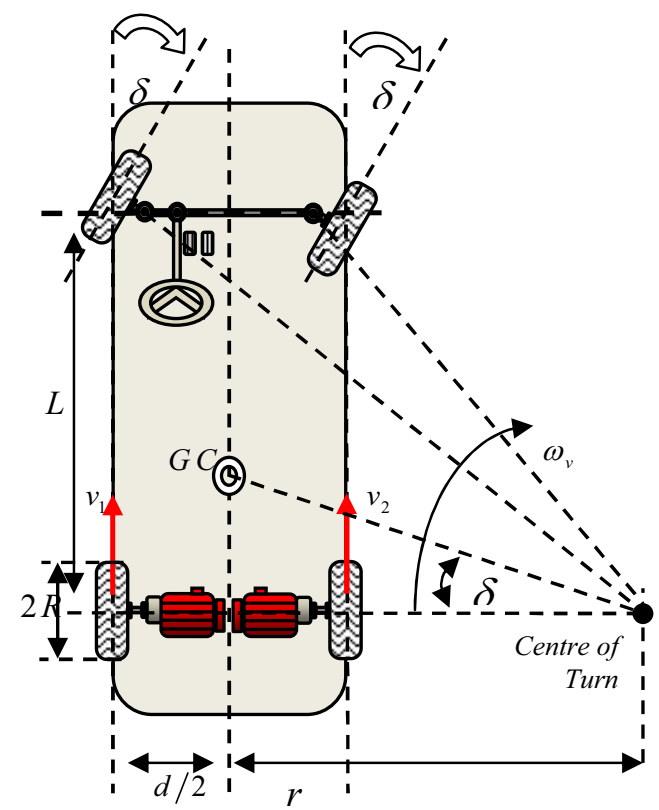

a

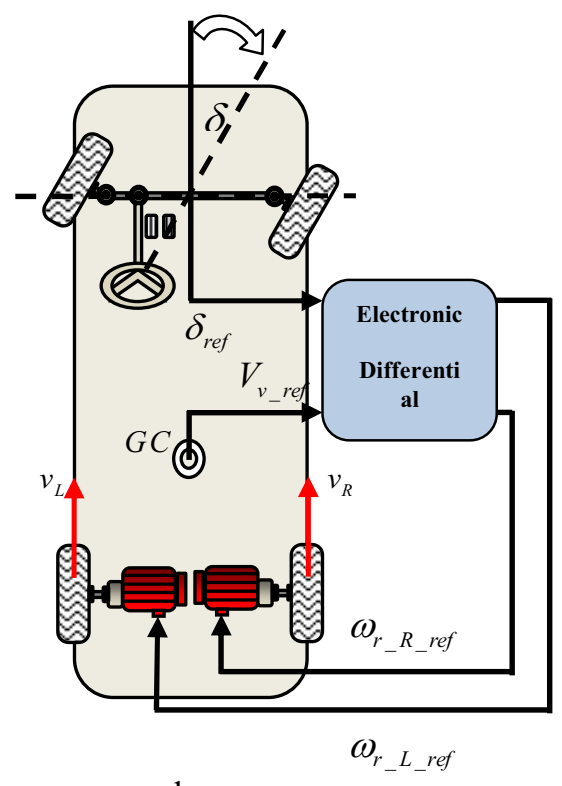

b

Fig.2 . Design model for vehicle structure driven. 


\section{Theory of the Control Used in the System}

Fig.3 shows scheme of the proposed control method of the electric traction system using the principle of the cascade control method, the structure comprises a speed control loop which generates the current reference $i_{\text {qref }}$ which imposes the control $v_{\text {qref }}$, the control $v_{\text {dref }}$ is imposed by the current regulation $i_{\text {dref }}$

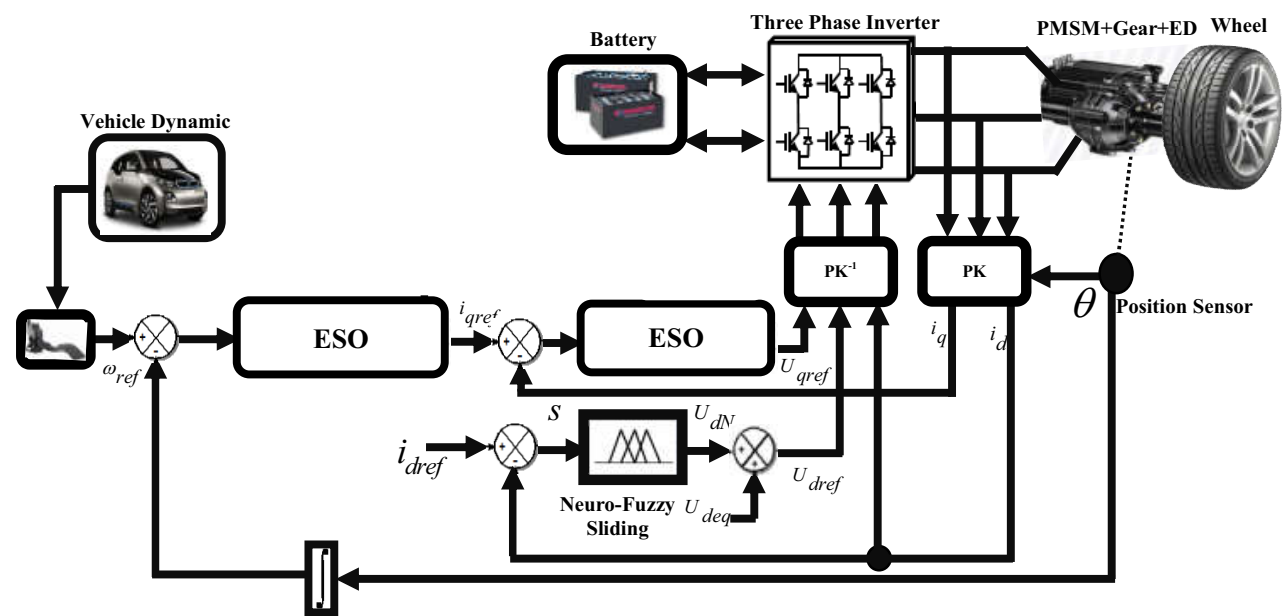

Fig. 3. Block diagram of the proposed control method for the Electric Vehicle

\subsection{Adaptive Neuro-Fuzzy Sliding Mode Controller}

The phenomenon of sliding modes could appear in a dynamic system restrained by ordinary differential equations with discontinuous right-hand sides. This phenomenon may happen if the control of the system state switches at high frequency.

However, like anything else the SMC has its own disadvantages, that is to say chattering phenomenon, which initiated from the interaction between parasitic dynamics and high frequency switching control. As stated in [15] and [23] several control methods were suggested in literature to prevent this problem.

We take the general equation suggested by J.J.Slotine to determine the sliding surface :

$s(x, t)=\left(\frac{\partial}{\partial t}+\lambda\right)^{(n-1)} . e$

$s$ : the sliding surface

$e:$ Error of the quantity to be controlled,

$\lambda$ : Vector of slopes of the $s$,

${ }_{n}$ : Relative degree, equal to the number of times it derives the output for the command to appear.

Sliding mode control of the electric traction system applies the principle of the cascade control method, the structure includes a speed control loop which generates the current reference $i_{\text {qref }}$ which imposes the control $v_{\text {qref }}$, the control $v_{\text {dref }}$ is imposed by the current regulation $i_{\text {dref }}$. 
During the sliding mode on direct current loop we have $s^{\bullet}\left(i_{d}\right)=0, U_{d N}=0$

$$
\begin{aligned}
& U_{\text {deq }}=\left(i_{\text {dref }}^{\bullet}+\frac{R_{s}}{L_{d}} i_{d}-\frac{L_{q}}{L_{d}} p \omega_{r} i_{q}\right) L_{d} \\
& U_{d N}=K_{d} \operatorname{sign}\left(s\left(i_{d}\right)\right)
\end{aligned}
$$

Where : $k_{d}$ is positive gain for the direct current regulator.

The proposed Neuro-fuzzy-sliding mode control (NFSMC) replaces the sign function of the direct current surface. Among many, the ANFIS (Adaptive Neuro Fuzzy Inference System) is typically architectured as demonstrated in Fig. 4 where a circle indicates a fixed node, however a square indicates an adaptive node. To simplify things, we consider two inputs $\mathrm{x}, \mathrm{y}$ and one output $\mathrm{z}$ among plenty FIS models [23], the Sugeno fuzzy model is the most widely applied. First due to its high interpretability, second computational efficiency, and built-in optimal and adaptive techniques as founded in [21].

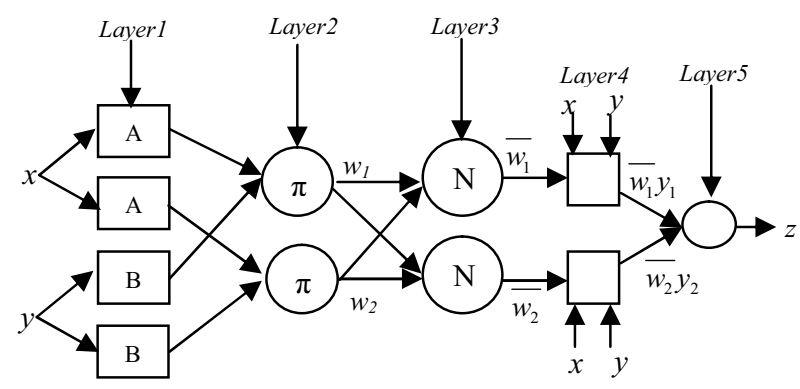

Fig. 4. Architecture of ANFIS

based on direct current error $(e)$ defined as [23]:

$$
e=i_{\text {dref }}-i_{d}
$$

Where $i_{d r e f}$ and $i_{d}$ are the references and the actual direct current, respectively.

In this study first order Sugeno type fuzzy inference was used for ANFIS and the typical fuzzy rule is:

if $e$ is $\mathrm{Ai}$ and $d e$ is $\mathrm{Bi}$ then $\mathrm{y}=\mathrm{f}(e)$.

Where $\mathrm{A}$ and $\mathrm{B}$ are fuzzy sets in the antecedent and $\mathrm{y}=\mathrm{f}(e)$ is a crisp function in the consequent.

The significances of ANFIS structure are:

Layer 1: Each adaptive node in this layer generates the membership grades for the input vectors $\mathrm{Ai}, \mathrm{i}=1, \ldots, 3$. In this paper, the node function is a triangular membership function:

$$
O_{i}^{1}=\mu_{A i}(e)=\left\{\begin{array}{lc}
0 & e \leq a_{i} \\
\frac{e-a_{i}}{b_{i}-a_{i}} & a_{i} \leq e \leq b_{i} \\
\frac{c-e}{c-b_{i}} & b_{i} \leq e \leq c_{i} \\
0 & c_{i} \leq e
\end{array}\right.
$$


Layer 2: The total number of rule is 9 in this layer. Each node output represents the activation level of a rule:

$O_{i}^{1}=w_{i}=\min \left(\left(\mu_{A i}(e)\right) \quad i=1 \ldots .3\right.$

layer 3:Fixed node $i$ in this layer calculate the ratio of the $i$-th rule's activation level to the total of all activation level:

$$
O_{i}^{3}=\overline{w_{i}}=\frac{w}{\sum_{j=1}^{n} w_{j}}
$$

Layer 4: Adaptive node $\mathrm{i}$ in this layer calculate the contribution of $\mathrm{i}$-th rule towards the overall output, with the following node function:

$O_{i}^{4}=\overline{w_{i}} y_{i}=\overline{w_{i}}\left(p_{i} e+r_{i}\right)$

Layer 5: The single fixed node in this layer computes the overall output as the summation of contribution from each rule:

$$
O_{i}^{5}=\sum_{i=1}^{2} \bar{w}_{i} y_{i}=\frac{w_{1} y_{1}+w_{2} y_{2}}{w_{1}+w_{2}}
$$

The parameters to be trained are, ai, bi, and ci of the premise parameters and pi and ri of the consequent parameters. Training algorithm requires a training set defined between inputs and output [23].

\subsection{Extended State Observer Controller}

Parameters variation, back electromotive forces, torque ripples, friction forces, unmodeled dynamics, external disturbance and load disturbances. All these different kinds of disturbances appear in real applications of electric vehicle based on PMSM motors. If the controller does not have adequate ability to these disturbances, they may demean the performance of the electric vehicle system.

Since the control structure that has been employed here is of cascade control loops for the speed regulation problem, the control scheme includes a speed loop and two current loops. For the d-axis current loop, Adaptif Neuro Fuzzy Sliding control algorithm is employed, for the speed loop and q-axis current loop, in order to estimate the different disturbances in each loop correspondingly, two ESO's are being introduced.

The lumped disturbances of the system which are composed of internal dynamics and external disturbances are considered as new state of the system by Extended State Observer (ESO) [18], where both states and lumped disturbances $a(t)$ can be estimated by this observer.

The construction of ESO will be shown below :

$$
x^{\bullet}=f(x, t)+d(t)+b u(t)
$$

where $f(x, t)$ is the unknown nonlinear function, $d(t)$ is the external disturbance, $u$ is the control signal, $x$ is the output of system, $b$ is the control gain. Considering the lumped disturbance $a(t)=f(x, t)+d(t)$ as an extended state, then Eq. (29) can be expressed as:

$$
x^{\bullet}=a(t)+b u(t)
$$

Define $x_{1}=x, x_{2}=a(t)$. The system (30) can be rearranged as:

$\left\{\begin{array}{l}x_{1}^{\bullet}=x_{2}+b u(t) \\ x_{2}^{\bullet}=c(t)\end{array}\right.$ 
where $c(t)=a^{\bullet}(t)$. And the ESO can be constructed as follows [26]:

$$
\left\{\begin{array}{l}
z_{1}^{\bullet}=z_{2}+2 P\left(z_{1}-\omega_{r}\right)+b u(t) \\
z_{2}^{\cdot}=-P^{2}\left(z_{1}-\omega_{r}\right)
\end{array}\right.
$$

where $-p(p>0)$ is the desired double pole of ESO. $z_{1}$ is the estimate of $\mathrm{x} 1$ and $z_{2}$ is the estimate of $a(t)$. Here, according to the analysis in [26], $z_{1}(t)$ is an estimate of the output $x(t)$ while $z_{2}(t)$ is an estimate of the lumped disturbance $a(t)$,i.e., $z_{1}(t) \rightarrow y(t)$ and $z_{2}(t) \rightarrow a(t)$.

A composite control law is designed as follows:

$u=u_{0}-\frac{z_{2}}{b}, u_{0}=k\left(x_{r e f}-z_{1}\right)$

where $x_{r e f}$ is the reference input of $x$.

\subsubsection{ESO Speed controller}

ESO-based controller for the speed loop is designed as follows:

The torque equation of PMSM system is:

$\omega_{r}^{\bullet}=\frac{3 p \psi_{f}}{2 J} i_{q}-\frac{f}{J} \omega_{r}-\frac{C_{r}}{J}=b i_{q r e f}+a(t)$

Where $b=\frac{3 p \psi_{f}}{2 J}$ is the control gain, $a(t)=\frac{3 p \psi_{f}}{2 J}\left(i_{q}-i_{q r e f}\right)-\frac{f}{J} \omega_{r}-\frac{C_{r}}{J}$ is the lumped disturbances consisting of the friction, the external load disturbances, and the tracking error of current-loop of $i_{q}$.

Then an ESO can be designed as:

$\left\{\begin{array}{l}z_{1}^{\cdot}=z_{2}+2 P\left(z_{1}-\omega_{r}\right)+b u(t) \\ z_{2}^{\cdot}=-P^{2}\left(z_{1}-\omega_{r}\right)\end{array}\right.$

where $z_{1}$ is the estimate of angular velocity $x, z_{2}$ is the estimate of the lumped disturbances.

A composite control law of speed loop can be designed as:

$$
i_{\text {qref }}=u=u_{0}-\frac{z_{2}}{b}, u_{0}=k\left(\omega_{\text {rref }}-\omega_{r}\right)
$$

where $k$ is the proportional gain, $\omega_{\text {rref }}$ is the reference speed, $i_{q}$ is the output of speed loop controller.

The block diagram of the ESO-based speed controller is shown in Fig.5. Note that the "generalized System" represents the two current loops which include PMSM and other components the same as that of Fig.3.

\subsubsection{ESO Iq Current controller}

The ESO of q-axis current loop can be designed as follows:

$i_{q}^{\bullet}=-p \omega_{r} i_{d}-\frac{R_{s}}{L} i_{q}-\frac{p \psi_{f}}{L} \omega_{r}+\frac{u_{q}}{L}$ 
$i_{q}=a_{q}(t)+\frac{u_{q}}{L}$

the lumped disturbance of q-axis current loop are $a_{q}=-p \omega_{r} i_{d}-\frac{R_{s}}{L} i_{q}-\frac{p \psi_{f}}{L} \omega_{r}$ consisting of the coupling between rotor speed and d-axis currents, the dynamics of q-axis current, the back electromotive force .

$$
\left\{\begin{array}{l}
z_{11}^{\bullet}=z_{12}+2 P_{q}\left(z_{11}-i_{q}\right)+\frac{u_{q}}{L} \\
z_{12}^{\bullet}=-P_{q}^{2}\left(z_{11}-i_{q}\right)
\end{array}\right.
$$

Where $-P_{q}\left(P_{q} \succ 0\right)$ is the desired double pole, $z_{11}$ is the estimated of $i_{q}$, and $z_{12}$ is the estimated of $a_{q}(t)$.

A hybrid control law of q-axis current loop can be designed as :

$$
u_{q}=u_{q 0}-L z_{12}, u_{q 0}=k_{q}\left(i_{q r e f}-i_{q}\right)
$$

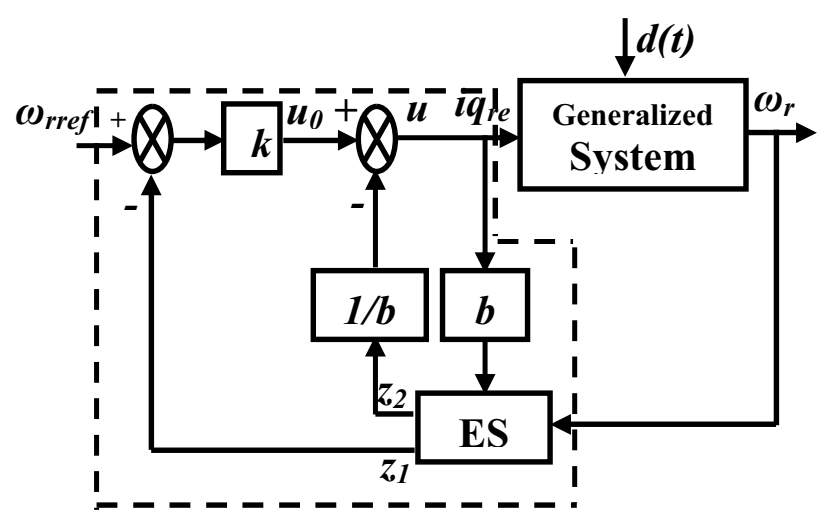

Fig.5. The bloc diagram of ESO-based speed controller

\section{Simulation Results}

It is important to note that the simulation requested by the driver, which demoes the wheel's speed while steering for $0<\mathrm{t}<481$ run under a Japanese urban driving cycle 11 mode 4.

With the vehicle approaching a right turn at $62 \mathrm{~s}$ (positive steering angle) and a left turn at $180 \mathrm{~s}$ (negative steering angle). It is evident that the electric vehicle adequately functioning according to the complicated series of accelerations, decelerations, and frequent stops.

Concerning the figure 6 and 7 , the vehicle is turning right at $t=62 \mathrm{~s}$, the speed left motor increases and right speed motor decreases, but the torque of the right wheel is increasing negatively which is basically a braking mode. As we can observe that the torque is returning to its initial value at the moment the speed of the right wheel is stabilized, where this working phase can be exploited for energy recuperation.

As we can view, the driver uses an inverse steering angle on the front of wheels at $t=$ $67 \mathrm{~s}$ in order to exit the curved section of the road. The electronic differential subsequently acts to equalize the speed of the drive motors; the speed difference of the driving wheels is shown in figure 6 . Unlike that on the right, the electronic differential is acting at once on 
both electric motors by lowering the speed of the left wheel, thus the vehicle approaching a left turn at $\mathrm{t}=180 \mathrm{~s}$. This speed is perceived in figure 6 .
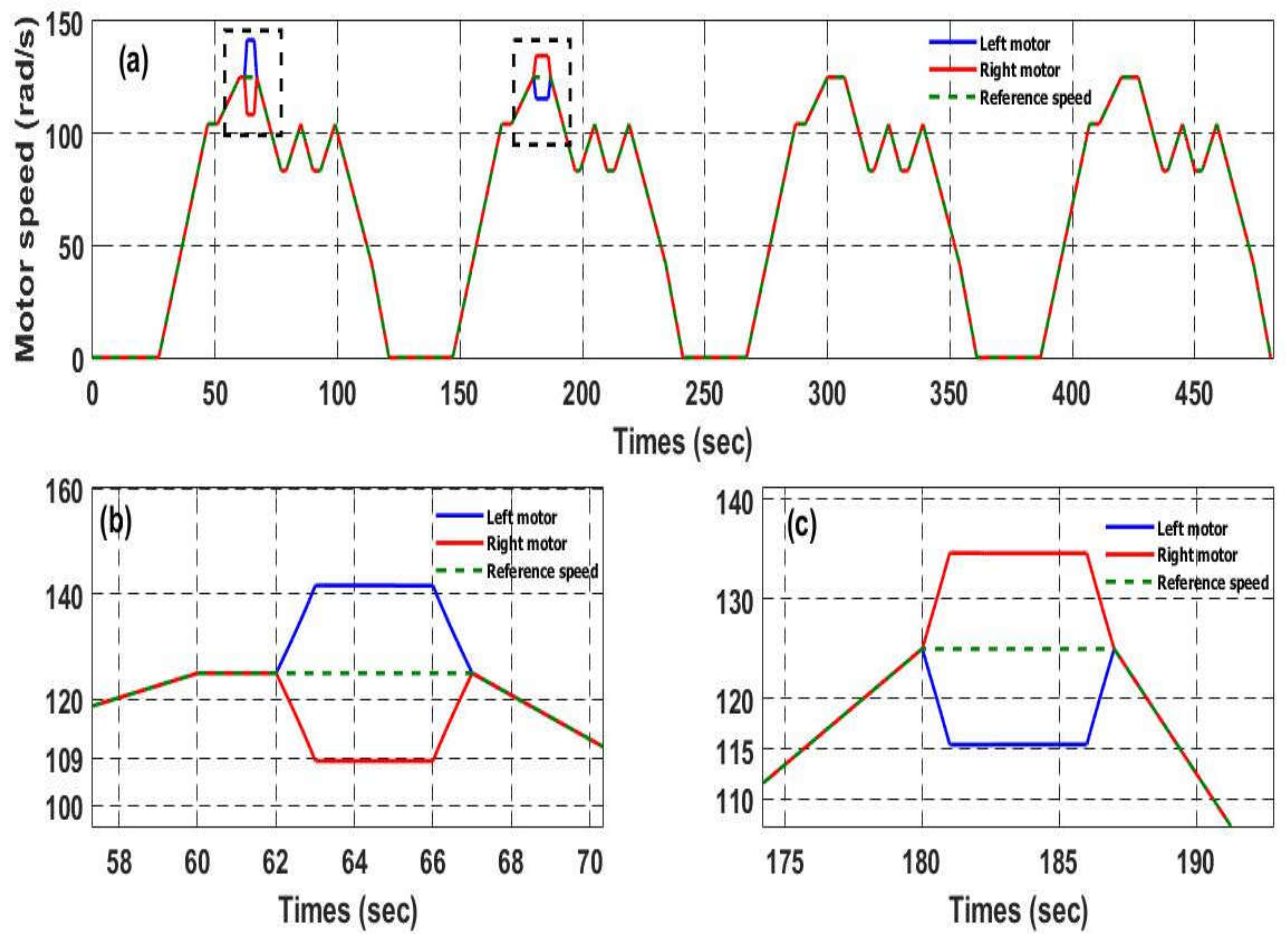

Fig. 6. (a): Speed motor of the vehicle, (b): zoom around $65 \mathrm{~s}$,(c):zoom around 186s
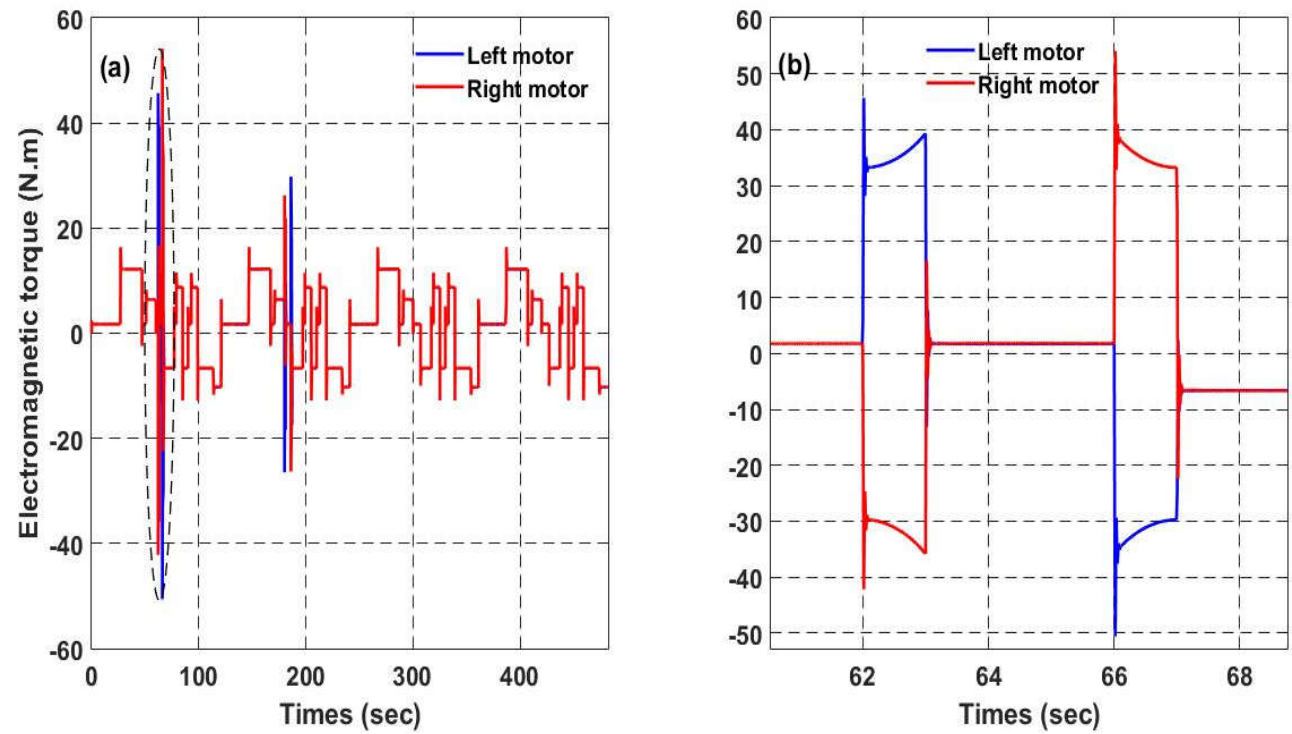

Fig. 7. (a):Variation of electromagnetic torque of the right and left motor in different phases,(b): zoom around $65 \mathrm{~s}$ 


\section{CONCLUSION}

In this paper, an Electronic Differential And Neuro-Fuzzy Sliding Mode Control With Extended State Observer For An Electric Vehicle System has been designed. The EV stability can be hard to manage particularly while cornering or even under slippery road condition. For that purpose, the recommended Electronic Differential (ED) has been developed. the problem of chattering phenomena in the PMSM electric motor had been eliminated by employing of tow types of controllers, neuro-fuzzy sliding mode controller on the direct current loop, ESO controller on the speed and quadratic current loops; taking into account the dynamic of the vehicle.T he simulations results indicate on the one hand that the traction system using NFSMC with ESO exhibits better dynamic performance, static performance, and robustness than the system with the traditional SMO, NFSMC with ESO has smaller chattering, higher accuracy, and no phase delay; and on other hands the electronic differential controls the driving wheels speeds with high accuracy either in flat roads or curved ones. In future studies, the proposed design technique will be extended to the improved ESO control strategy of current and position controls.

\section{Referencesm}

1. Michele D G, Elena P, Giorgio M , Energy Feb.2015;82:294-311.

2. Kandasamy N K , King J T , Energy Jun.2016;111:190-196.

3. A. Roque, J.Esteves, J.Maia, P.Verdelho, Proceeding of Electrimacs 99 September (1999)

4. M. Martin, Info Tech $n^{\circ} 13$, June (2004)

5. K. Houacine, R. Mellah and S. Guermah, International Journal of Electric and Hybrid Vehicles, vol. 7, pp. 189-207, 2015.

6. S. Morimoto et al., IEEE Transactions on Industrial Electronics, vol.61, no.10, pp. 5749-5756, Oct. 2014.

7. A. Sant and K. Rajagopal, IEEE Transactions on Magnetics, vol.45, no.10, , pp. 4672-4675, Oct. 2009.

8. Golea N, Golea A, Kadjoudy M. IEEE international conference on industrial technology. Mumbai, India; 2006.p p. 1533-7.

9. Hsien TL, Sun YY, Tai MC, IEE Proc - Electr Power Appl 1997;144(3):173-81.

10. Wai RJ. , IEEE Trans Indust Electron 2001;8(5):926-44.

11. Wang GJ, Fong CT, Chang KJ. , IEEE Trans Indust Electron 2001;48(2):408-15.

12. Kung YS, Tsai MH., IEEE Trans Power Electron 2007;22(6):2476-86.

13. Max Reitz, Xin Wang, Patrick Gu, 978-1-5090-0403-4/16,2016,IEEE.

14. Chung, S.C.Y., Lin, C.L., IEEE Trans. Automatic Control 44, 563-568 (1999).

15. N.K. Yadav and R. K. Singh , International Journal of Artificial Intelligence \& Applications, Vol. 4, July (2013).

16. L. Jian, Z. Wang, L. Jian, Z. Wang, Microcomputer Information, Vol. 23, n . 9, pp. 22-24, (2007)

17. H. Bai, R. Qi, Transactions of China Electrotechnical Society, Vol. 23, n. 8, pp. 3640, (2008)

18. J. Q. Han, Control Decision, vol. 10, pp. 85-88, 1995, (in Chinese).

19. C.C. Chan, Y.S. Wong, IEEE Power and Energy Magazine, Vol. 2, No. 6, Nov/Dec. (2004), pp. $24-33$.

20. R.F. Fung, C.L. Chiang, G.C. Wu, Appl. Math Model. 34 (2010) 2323-2335 
21. J.S.R. Jang, IEEE Trans Syst. Man. Cybernet, 1993; 23(3), pp. 665-8

22. D. Foito, A. Roque, J.Esteves, J.Maia, Proceeding of EVS17, Montreal , October (2000)

23. A.Tahour, A.Hamza, A.Aissaoui, Advance in Electrical and Computer Engineering, Vol,8.15,Number 1(29),(2008)

24. A.Tahour, H.Abid, A.Aissaoui, serbian journal of electrical engineering Vol. 4, No. 1, June (2007), 23-34

25. Sadeghi, S. \& Mirsalim, M, International peer-reviewed scientific journal of Applied sciences, Vol.7, No.1, (2010), pp. 51-71, ISBN 1785-8860.

26. Miklosovic R, Gao ZQ, In: 39th IAS annual meeting on industry application conference; 2004. p. $1495-502$. 